\section{New Drug Approvals}

Cannabidiol (Oral Solution)

Company: GW Pharmaceuticals plc

Date of Approval: June 25, 2018

Epidiolex (cannabidiol) is a prescription pharmaceutical formulation of highly-purified, marijuana plant-derived cannabidiol (CBD) for the treatment of seizures associated with Lennox-Gastaut syndrome or Dravet syndrome in patients two years of age or older.

\section{Desmopressin Acetate (Sublingual Tablets)}

Company: Ferring Pharmaceuticals Inc.

Date of Approval: June 21, 2018

Nocdurna (desmopressin acetate) is a vasopressin analog indicated for the treatment of nocturia due to nocturnal polyuria in adults.

\section{Moxidectin Tablets}

Company: Medicines Development for Global Health Date of Approval: June 13, 2018

Moxidectin is a macrocyclic lactone anthelmintic medicine indicated for the treatment of river blindness (onchocerciasis) in patients aged 12 years and older.

\section{Pegfilgrastim-jmdb (Injection)}

Company: Mylan N.V.

Date of Approval: June 4, 2018

Fulphila (pegfilgrastim-jmbd) is a leukocyte growth factor biosimilar to Neulasta (pegfilgrastim) indicated to reduce the duration of febrile neutropenia in patients treated with chemotherapy.

\section{Baricitinib (Tablets)}

Company: Eli Lilly and Company

Date of Approval: May 31, 2018

Olumiant (baricitinib) is a Janus kinase (JAK) inhibitor for the treatment of rheumatoid arthritis.
Amlodipine and Celecoxib (Tablets - formerly KIT-302)

Company: Kitov Pharma Ltd.

Date of Approval: May 31, 2018

Consensi (amlodipine and celecoxib) is a calcium channel blocker and nonsteroidal anti-inflammatory drug combination for the treatment of both hypertension and pain associated with osteoarthritis.

\section{Estradiol (Vaginal Inserts)}

Company: TherapeuticsMD, Inc.

Date of Approval: May 29, 2018

Imvexxy (estradiol) is a bio-identical 17 $\beta$-estradiol vaginal insert for the treatment of dyspareunia (vaginal pain during sexual intercourse) due to menopause.

\section{Pegvaliase-pqpz (Injection)}

Company: BioMarin Pharmaceutical Inc.

Date of Approval: May 24, 2018

Palynziq (pegvaliase-pqpz) is a phenylalaninemetabolizing enzyme indicated to reduce blood phenylalanine concentrations in adult patients with phenylketonuria.

\section{Abiraterone Acetate (Tablets)}

Company: Sun Pharmaceutical Industries Inc.

Date of Approval: May 22, 2018

Yonsa (abiraterone acetate) is an ultramicrosize formulation of the oral CYP17 inhibitor abiraterone acetate (approved as Zytiga) used in combination with methylprednisolone for the treatment of metastatic castration-resistant prostate cancer.

\section{Avatrombopag (Tablets) \\ Company: Dova Pharmaceuticals, Inc. \\ Date of Approval: May 21, 2018}

Doptelet (avatrombopag) is a second generation, orally administered thrombopoietin receptor agonist (TPO-RA) indicated for the treatment of thrombocytopenia in patients with chronic liver 
disease who are scheduled to undergo a medical procedure.

\section{Sodium Zirconium Cyclosilicate (Oral Suspension) \\ Company: AstraZeneca \\ Date of Approval: May 18, 2018}

Lokelma (sodium zirconium cyclosilicate) is a potassium binder indicated for the treatment of hyperkalemia in adults.

\section{Erenumab-Aooe (Injection)}

Company: Amgen Inc.

Date of Approval: May 17, 2018

Aimovig (erenumab-aooe) is a calcitonin generelated peptide (CGRP) receptor antagonist indicated for the preventive treatment of migraine.

lofexidine Hydrochloride (Tablets)

Company: US WorldMeds

Date of Approval: May 16, 2018

Lucemyra (lofexidine hydrochloride) is a selective $\alpha$-2-adrenergic receptor agonist indicated for reducing the severity of withdrawal symptoms in patients experiencing opioid withdrawal.

\section{Epoetin Alfa-Epbx (Injection)}

Company: Hospira, Inc.

Date of Approval: May 15, 2018

Retacrit (epoetin alfa-epbx) is an erythropoiesisstimulating agent (ESA) biosimilar to Epogen/Procrit (epoetin alfa) indicated for treatment of anemia caused by chronic kidney disease, chemotherapy, or use of zidovudine in patients with HIV infection. Retacrit is also approved for use before and after surgery to reduce the chance that red blood cell transfusions will be needed because of blood loss during surgery.

\section{Polyethylene glycol 3350 with electrolytes (Oral Solution) \\ Company: Salix Pharmaceuticals, Inc. \\ Date of Approval: May 4, 2018}

Plenvu (polyethylene glycol 3350, sodium ascorbate, sodium sulfate, ascorbic acid, sodium chloride and potassium chloride) is a lower-volume, polyethylene glycol based osmotic laxative indicated for cleansing of the colon (bowel preparation) prior to colonoscopy.

Coagulation factor Xa (recombinant), inactivatedzhzo

Company: Portola Pharmaceuticals, Inc.

Date of Approval: May 3, 2018

Andexxa (coagulation factor $\mathrm{Xa}$ (recombinant), inactivated-zhzo) is a recombinant modified human Factor $\mathrm{Xa}(\mathrm{FXa})$ protein indicated for patients treated with rivaroxaban and apixaban, when reversal of anticoagulation is needed due to life-threatening or uncontrolled bleeding.

Tolvaptan (Tablets)

Company: Otsuka Pharmaceutical Co., Ltd

Date of Approval: April 23, 2018

Jynarque (tolvaptan) is a selective vasopressin $\mathrm{V}_{2^{-}}$ receptor antagonist indicated to slow kidney function decline in adults at risk of rapidly progressing autosomal dominant polycystic kidney disease (ADPKD).

Fosnetupitant and palonosetron

Company: Helsinn Healthcare SA

Date of Approval: April 19, 2018

Akynzeo for Injection (fosnetupitant and palonosetron) is a substance P/neurokinin-1 (NK-1) receptor antagonist and serotonin-3 (5-HT3) receptor antagonist combination indicated for use with dexamethasone for the prevention of chemotherapyinduced nausea and vomiting (CINV).

\section{Burosumab-twza (Injection)}

Company: Ultragenyx Pharmaceutical Inc.

Date of Approval: April 17, 2018

Crysvita (burosumab-twza) is a fibroblast growth factor 23 (FGF23) blocking antibody for the treatment of $x$-linked hypophosphatemia (XLH). 


\section{Fostamatinib (Tablets)}

Company: Rigel Pharmaceuticals, Inc.

Date of Approval: April 17, 2018

Tavalisse (fostamatinib) is an oral spleen tyrosine kinase (SYK) inhibitor for the treatment of patients with chronic immune thrombocytopenia (ITP).

\section{Ibalizumab-uiyk (Injection)}

Date of Approval: March 6, 2018

Company: TaiMed Biologics USA Corp.

Trogarzo (ibalizumab-uiyk) is a CD4-directed postattachment HIV-1 inhibitor for the treatment of multidrug resistant human immunodeficiency virus-1 (HIV-1) infection in heavily treatment-experienced adults.

\section{Tildrakizumab-asmn (Injection)}

Date of Approval: March 20, 2018

Company: Sun Pharmaceutical Industries Ltd.

Ilumya (tildrakizumab-asmn) is a humanized, antiIL-23p19 monoclonal antibody for the treatment of moderate-to-severe plaque psoriasis.

Information Collected and complied by:

Md. Akbar Hossain

Department of Pharmacy

ASA University Bangladesh
Efavirenz, lamivudine and tenofovir disoproxil fumarate (Tablets)

Date of Approval: March 22, 2018

Company: Mylan N.V.

Symfi (efavirenz, lamivudine and tenofovir disoproxil fumarate) is a three-drug combination of a non-nucleoside reverse transcriptase inhibitor (efavirenz), and two nucleo(t)side reverse transcriptase inhibitors (lamivudine and tenofovir disoproxil fumarate) indicated as a complete regimen for the treatment of human immunodeficiency virus type 1 (HIV-1) infection.

\section{Source: Drugs.com}

